\title{
Interaction of young stellar object jets with their accretion disk $\star, \star \star$
}

\begin{abstract}
N. Soker
Department of Physics, Technion-Israel Institute of Technology, 32000 Haifa, Israel

e-mail: soker@physics.technion.ac.il

Received 21 October 2004 / Accepted 10 January 2005

Abstract. I reexamine recent observations of velocity profiles across jets blown by young stellar objects, and argue that the observations do not support the earlier interpretation of jets rotating around their symmetry axes. Instead, I propose that interaction of the jets with a twisted-tilted (wrapped) accretion disk can form the observed asymmetry in the jets' line of sight velocity profiles. The proposed scenario is based on two plausible assumptions. (1) There is an inclination between the jet and the outer parts of the disk, such that the jet is perpendicular to the inner part of the disk; i.e., there is a twisted-tilted (wrapped) disk. (2) The disk-jet interaction slows the jet down as the jet entrains mass from the disk, with larger decelaration of jet segments closer to the tilted disk. The proposed scenario can account for the basic properties of the observed velocity profiles, while offering the advantage of not needing to refer to any magnetic jet launching model, and there is no need to invoke jet rotation with a huge amount of angular momentum.
\end{abstract}

Key words. accretion, accretion disks - ISM: jets and outflows - stars: pre-main-sequence - stars: formation

\section{Introduction}

Asymmetry in the line of sight velocity across some jets ejected by young stellar objects (YSOs) has been interpreted as caused by a large scale rotation of the material in each jet around the jet's symmetry axis (e.g., Bacciotti et al. 2002; Coffey et al. 2004, hereafter B2002 and C2004). Rotation around the jet axis is predicted by the magneto-centrifugal models for jet launching. In these models the magnetic fields that are anchored into the accretion disk-star system play a dominate role in accelerating the jet's material from the accretion disk. Most models in YSOs are based on the operation of large scale magnetic fields driving the flow from the disk: either via the "centrifugal wind" mechanism, first proposed by Blandford \& Payne (1982), or from a narrow region in the magnetopause of the stellar field via an "X-wind mechanism" introduced by Shu et al. (1988, 1991) and, in a somewhat different setting, by Ferreira \& Pelletier (1993, 1995). See recent reviews and papers by Königl and Pudritz (2000), Shu et al. (2000), Ferreira (2002), and Krasnopolsky et al. (2003). It should, however, be pointed out that there are still open key issues (e.g. Heyvaerts et al. 1996). One of them is the origin of the large scale magnetic fields and the manner open field lines of sufficiently strong magnitude persist (in the centrifugal wind models). Another open issue is the manner by which a stellar field interacts with the disk, allowing inflow and at the same time driving an outflow (in the

\footnotetext{
* Research supported by the Israel Science Foundation.

$\star \star$ Appendix is only available in electronic form at http://www.edpsciences.org
}

$\mathrm{X}$-wind models). In addition, it seems that thermal pressure is needed in some of these models (e.g., Ferreira \& Casse 2004).

In a recent paper, Cerqueira \& de Gouveia Dal Pino (2004) conduct magnetohydrodynamics (MHD) 3D numerical simulations of YSO jets with rotation. They use parameters suitable for the jet of DG Tauri, a YSO studied by B2002, and obtain radial velocity maps in good agreement with the observations of B2002. In the Appendix I point out some difficulties with this interpretation of jet rotation.

Magnetic fields might also play a role in triggering the jet's ejection event, e.g., by causing instabilities in the disk and in collimating the jets (e.g., Heyvaerts \& Norman 1989). The collimation issue is, however, still quite controversial, and while magnetic collimation is certainly plausible, its exact nature is probably quite involved and still not fully understood (see the recent works of Bogovalov \& Tsinganos 2001; Li 2002). In some models external pressure is required to maintain collimation, e.g., Kato et al. (2004) and the magnetic tower model of Lynden-Bell (2003). Although some of the arguments in these papers apply to relativistic jets, I mention them here in my search for a unified collimation machanism in different jets' environments. In Sect. 2 I discuss some aspects of disk-jet interaction in YSOs.

In some models, magnetic fields do not play a major role in launching the jet. These include the thermally-launched jet model (Torbett 1984; Torbett \& Gilden 1992) that was applied to YSOs (Soker \& Regev 2003) and cataclysmic variables (Soker \& Lasota 2004). Motivated by non-magnetic models, in 
Sect. 3 I show that the asymmetric radial velocity field of these YSO jets may be interpreted in an alternative way, where the magnetic field play no major role. In this proposed model, instead of jet rotation, the velocity field is attributed to interaction of the jet with the accretion disk wind and/or corona. A summary of the main results is found in Sect. 4.

\section{Interaction of a fast outflow with the disk}

I here discuss an alternative explanation for the results in C2004, one based on the interaction of an outflow (jets), blown at large speeds from the vicinity of the central star, with the accretion disk, mainly via entrainment of disk mass by the jets. The entrainment process itself has been studied in the past. This entrainment is likely to occur in a turbulent mixing layer between the jet and the ambient gas (e.g., Kahn 1980; Canto \& Raga 1991). While most studies consider entrainment of (molecular) gas at distances of hundreds of AU from the source (e.g. Davis et al. 2004), I consider entrainment of disk material at a distance of several $\mathrm{AU}$ from the source. Based on $\mathrm{CO}$ observations, Stahler (1994) build an empirical model for entrainment in YSO jets on scales of hundreds of AU. According to this model, the velocity of matter inside the jet decreases with increasing distance from the jet's axis. This velocity profile is assumed here as well. The next step in developing the presently proposed scenario will be to include such an effect for interaction of a jet with a wrapped disk, but on a scale of several AU rather than hundreds of AU.

First I note that accretion disks around YSOs can be quite extended in the vertical direction (e.g., Semenov et al. 2004; Malbet et al. 2001). In addition, the disk may blow a slow wind from an extended region caused, e.g., by coronal type magnetic activity on the surface of the disk. I assume therefore that a fast wind, hereafter referred to as jets, blown from the vicinity of the central star at a speed of $v_{\mathrm{j}} \sim 300-500 \mathrm{~km} \mathrm{~s}^{-1}$, interacts with the disk and/or its wind. The outer parts of the jets are influenced the most. Let us first assume that the outskirts of the jet are strongly shocked at a distance of $r_{\mathrm{s}}$ from the central star. The density of the post-shocked gas, which is $\sim 4$ times the pre-shocked density, is given by

$$
\begin{aligned}
\rho_{\mathrm{s}} \simeq & 3 \times 10^{-19} \frac{\dot{M}_{\mathrm{j}}}{10^{-8} M_{\odot} \mathrm{yr}^{-1}}\left(\frac{r_{\mathrm{s}}}{10 \mathrm{AU}}\right)^{-2} \\
& \times\left(\frac{v_{\mathrm{j}}}{300 \mathrm{~km} \mathrm{~s}^{-1}}\right)^{-1} \mathrm{~g} \mathrm{~cm}^{-1}
\end{aligned}
$$

where $\dot{M}_{\mathrm{j}}$ is the mass loss rate into the two jets together. I assume above a spherically symmetric fast wind (jet); an initial (poor) collimation means a higher jet's density. On the other hand, higher mass loss rate near the jet axis (e.g., Liseau et al. 2005) means a lower density on the jet's outskirts. The density given in Eq. (1) is lower than the disk's surface density $\rho_{\mathrm{d}} \simeq 10^{-17} \mathrm{~g} \mathrm{~cm}^{-1}$ at $r_{\mathrm{d}}=10 \mathrm{AU}$ in the model of Semenov et al. (2004) for an accretion rate of $\dot{M}_{\mathrm{acc}}=10^{-7} M_{\odot} \mathrm{yr}^{-1}$. The gas shocked to a temperature of

$$
T_{\mathrm{s}}=\frac{3}{16} \frac{\mu m_{\mathrm{H}}}{k} v_{\mathrm{j}}^{2}=1.3 \times 10^{6}\left(\frac{v_{\mathrm{j}}}{300 \mathrm{~km} \mathrm{~s}^{-1}}\right)^{2} \mathrm{~K} .
$$

For this temperature I approximate the cooling curve (e.g., Gaetz et al. 1988) by $\Lambda=3.5 \times 10^{-23}\left(T / 10^{7} \mathrm{~K}\right)^{-1 / 2} \mathrm{erg} \mathrm{cm}^{3} \mathrm{~s}^{-1}$, for $2 \times 10^{5} \lesssim T \lesssim 2 \times 10^{7} \mathrm{~K}$. The cooling rate in power per unit volume is given by $\Lambda n_{\mathrm{e}} n_{\mathrm{p}}$, where $n_{\mathrm{e}}$ and $n_{\mathrm{p}}$ are the electron and proton density, respectively. I take the cooling time at a constant pressure $\tau_{\mathrm{c}}=(5 / 2) n k T / \Lambda n_{\mathrm{e}} n_{\mathrm{p}}$ for a fully ionized post-shock gas. For the flow time I take $\tau_{\mathrm{f}}=r_{\mathrm{s}} / v_{\mathrm{j}}$, which is about the time required for the high post shock pressure to reaccelerate the post shock gas. Relating the shock temperature to the speed of a fully ionized solar composition wind, and taking the density from Eq. (1), gives

$\chi \equiv \frac{\tau_{\mathrm{c}}}{\tau_{\mathrm{f}}} \simeq 10\left(\frac{\dot{M}_{\mathrm{j}}}{10^{-8} M_{\odot} \mathrm{yr}^{-1}}\right)^{-1}\left(\frac{r_{\mathrm{s}}}{10 \mathrm{AU}}\right)\left(\frac{v_{\mathrm{j}}}{300 \mathrm{~km} \mathrm{~s}^{-1}}\right)^{5}$.

The reacceleration distance is shorter than $r_{\mathrm{s}}$, and the reacceleration time shorter than $r_{\mathrm{s}} / v_{\mathrm{j}}$. Also, the shock is oblique, and less of the wind kinetic energy is thermalized. Therefore, the value given in Eq. (3) is a lower limit.

From Eqs. (1)-(3) the following conclusions can be drawn.

(1) The density in the surface layer of disks around YSOs can be higher than that in the jet, influencing its propagation. The main modes of interaction are thereby entraining of the disk's mass by the jets, a process that slows down the region of the jets entraining the mass, and deflection of the jet's outskirts via a strong shock, a process which recollimates the jet and which may deflect somewhat the jet.

(2) The shocked material is heated to soft X-ray emission temperatures and can cool on a longer time scale than the time it is being re-accelerated; that is, there is an adiabatic-type flow. Note that the fraction of the mass in the jet that is shocked to X-ray emitting temperature is very low, as only the outskirts of the jets are shocked. Pressure gradient then collimates the inner regions of the jet, and entrainment slows the jet down. Therefore, X-ray emission from the shocked region will be weak, which is compatible with the Chandra observations of Getman et al. (2002), who find that the presence or absence of an outflow does not appear to produce any difference in the X-ray properties of YSOs.

(3) The relatively small energy loss implies that the reacclerated gas reaches velocity close to its original speed.

(4) However, because of the high disk-density in the interaction region, even a small-volume mixing implies that substantial disk material is entrained by the jet outskirts. This, and some radiative cooling, will slow down the reaccelerated gas.

(5) The last point implies that if a large amount of gas from the disk is entrained by the fast jet, a slow dense jet might be formed.

(6) The inner boundary of the surface layer in the disk model of Semenov et al. (2004) mentioned above is at a height of $z_{U}=0.17\left(r_{\mathrm{d}} / \mathrm{AU}\right)^{1.27} \mathrm{AU}$. The outer boundary of the surface layer flares even faster. If the surface layer redirects the outskirts of the jets, this flaring will lead to collimation of the jet.

(7) Cooling time is not much longer than flow time. Because of inhomogeneities, some blobs may still cool. I attribute differences in the velocities observed in different lines by 


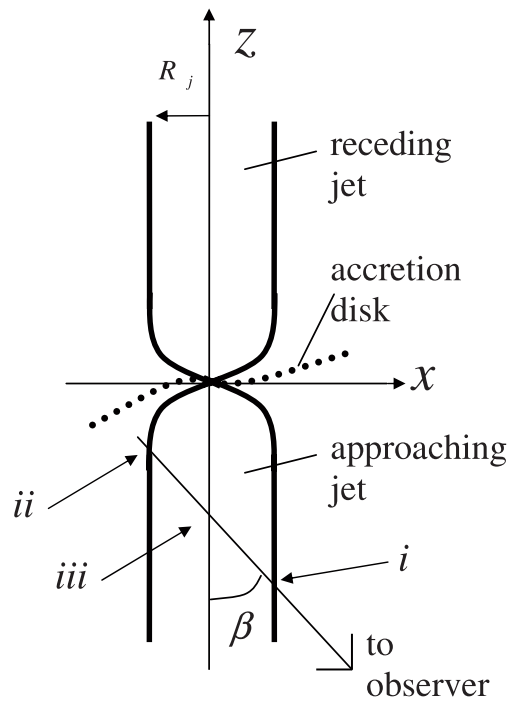

Fig. 1. Schematic drawing of the jet-disk-observer geometry in the plane defined by the jets' symmetry $(z)$ axis, and the line of sight. The solid thick lines mark the boundary of the two jets. The dotted thick line shows a cut through the twisted-tilted accretion disk. The three points which are marked $i, i i$, and $i i i$, are the three points along each line of sight, at distance $y$ from the jet's axis, for which the line of sight velocity is plotted in Fig. 3 .

C2004 to such inhomogeneities, which possibly led to thermal instabilities as well.

\section{Interaction with the disk and radial velocity profiles}

In the previous section I discussed the interaction of a fast wind ( 2 opposite ejected jets) with the accretion disk. This might result in jets' collimation, where the basic collimating force is thermal pressure, with possible help from ram pressure of a slow disk's wind. Magnetic hoop-stress might still play a role, but not necessarily a dominant one. In this section I show that in principle the radial-velocity gradient in the jets observed by B2002 and C2004 can be explained by interaction of the jets with the accretion disk, if one assumes that the accretion disk is tilted with respect to the jets' axis. What C2004 attributes to rotation, I attribute to a different degree in the slowing down process of different segments of the jets, caused by a tilted accretion disk. Since the jets will be blown perpendicular to the inner disk, what is required is for the inner disk to be twisted with respect to the further out regions of the disk. These are the outer parts of the disk which the jets interact with. Such a twisted (wrapped) disk can result from (i) radiation induced warping (Armitage \& Pringle 1997; see Figs. 7 and 10 of Wijers \& Pringle 1999 for the geometry of a twisted disk); (ii) from the influence a stellar binary companion (Larwood et al. 1996); or (iii) possibly from the influence of a massive planet.

The assumed disk structure is schematically drawn in Fig. 1. The $z$ axis is taken along the jet axis with the positive side in the receding (red-shifted) jet direction. The $x z$ plane is taken to be the plane defined by the jet axis and the line of sight with the positive $x$ axis on the observer side. The angle between the approaching (blue-shifted) jet's axis and the line

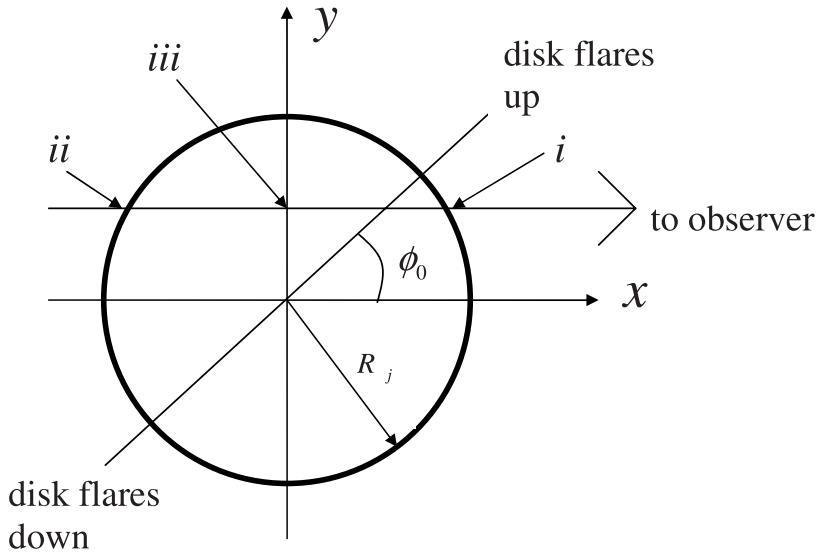

Fig. 2. Like Fig. 1, but showing the plane perpendicular to the jets' axis, and through the source of the jets. The tilting and twisting of the accretion disk is monotonic, with maximum flaring in the $+z$ direction at an angle $\phi_{0}$ to the $+x$ axis, and the maximum flaring in the $-z$ direction occurring at an angle $\pi+\phi_{0}$ to the $+x$ axis.

of sight is $\beta$. The observed section of the jet is assumed to be cylindrical with radius $R_{\mathrm{j}}$. The $y$ axis is perpendicular to the jet's axis and to the line of sight (Fig. 2). The first basic assumption is that the disk plane is inclined to the $x y$ plane; it has a maximum height toward the $+z$ direction at an angle $\phi_{0}$ from the $x$ axis, and a maximum height in the $-z$ direction at an angle of $\pi+\phi_{0}$ relative to the $x$ axis. The tilt angle is $\alpha$. The second basic assumption is that the disk slows down the jet with larger decelaration of jet segments closer to the tilted disk and less efficient deceleration closer to the jets' axis and away from the disk. I also consider all regions with jets far from their origins, after the different segments have reached their terminal speed; i.e., $v_{\mathrm{j}}(r, \phi)$ does not depend on $z$. I parameterize the slowing down of the jet material by taking the velocity at a distance $r$ from the jet axis and at an angle $\phi$ to the $x$ axis as

$\frac{\boldsymbol{v}_{\mathrm{j}}(r, \phi)}{v_{\mathrm{ja}}}=k \hat{z}-C_{1}\left[k+f_{2}(\alpha) \cos \left(\phi-\phi_{0}\right)\right]\left(\frac{r}{R_{\mathrm{j}}}\right)^{n} \hat{z}$,

where $C_{1}>0$, which is a constant, and $1>f_{2}(\alpha) \geq 0$, which is a monotonic function of the tilt angle $\alpha$, depend both on the strength of the jet-disk interaction; $v_{\mathrm{ja}}$ is the magnitude of the outflow speed along the jets' axis, and

$k \equiv\left\{\begin{array}{cl}1 & \text { for receding jet } \\ -1 & \text { for approaching jet. }\end{array}\right.$

The observation at specific point across the jet includes all jet segments along the line of sight to that point. To get a sense of the range of the observed velocities, I draw the component of the jet's radial velocity in Fig. 3 along the line of sight, as a function of the distance from the jet's axis for three locations along the line of sight for each point:

(i) The close (to the observer) side of the jet along the line of sight (marked $i$ in Figs. 1 and 2; thick solid line in Fig. 3). In this case the point is located at $(r, \sin \phi)=\left(R_{\mathrm{j}}, y / R_{\mathrm{j}}\right)$, with $\cos \phi=+\sqrt{1-y^{2} / R_{\mathrm{j}}^{2}}$. Substituting this value in Eq. (4) yields, for the jet velocity component along the 


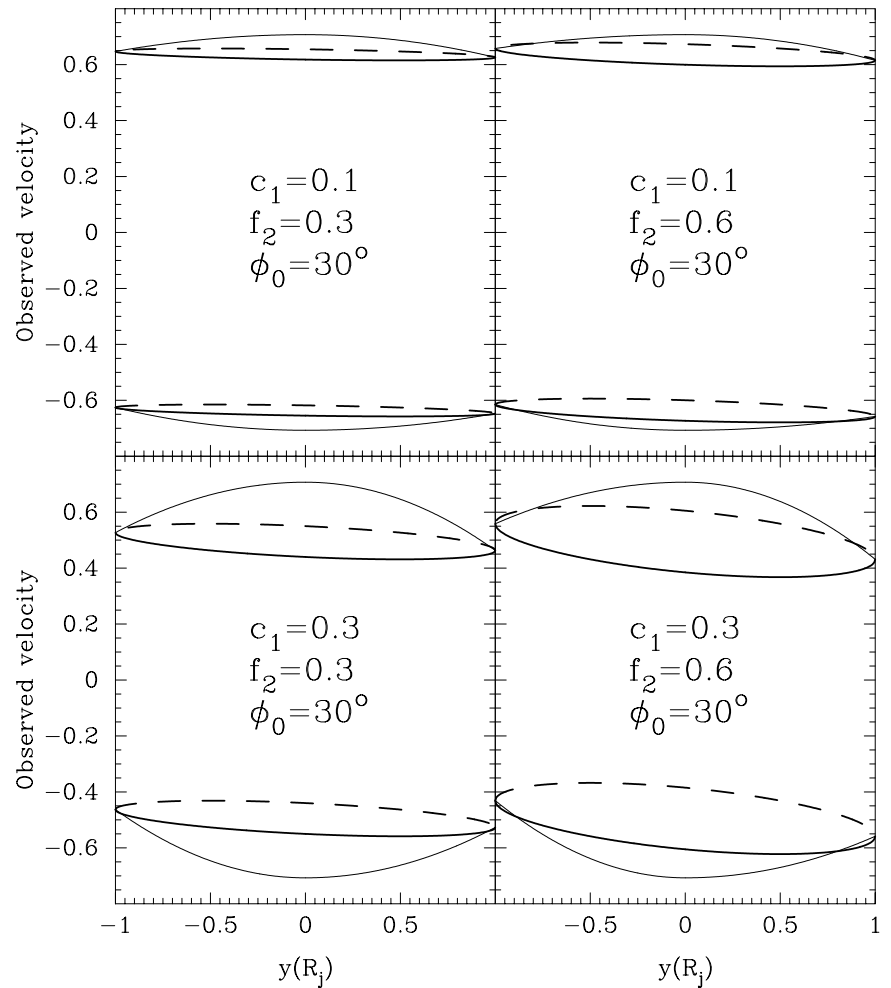

Fig. 3. The observed line of sight velocity across the jet, in units of the real velocity along the jet's axis. $y$ is the distance from the jet's symmetry axis, perpendicular to the line of sight. In each panel the upper 3 lines are for the receding jet, while the lower 3 lines are for the approaching jet. Thick solid line is the velocity of the closest point in the jet along the line of sight (Eq. (6); point $i$ in Figs. 1 and 2); Thick dashed line is the velocity of the farthest point in the jet along the line of sight (Eq. (6) with minus sign in front of the square root; point $i i$ in Figs. 1 and 2); Thin solid line is the velocity of the jet's element closest to the jet's axis, namely, on a line from the jet's axis and perpendicular to the line of sight (Eq. (7); point iii in Figs. 1 and 2). In all panels the jet axis in the $-z$ direction has an angle of $\beta=45^{\circ}$ to the line of sight. $\phi_{0}$ is defined in Fig. 2, while the meaning of $n, C_{1}$ and $f_{2}$ can be seen in Eq. (4); $n=2$ in all cases drawn.

line of sight:

$$
\begin{aligned}
\frac{v_{\mathrm{j}}}{v_{\mathrm{ja}}}= & {\left[k-C_{1} k-C_{1} f_{2}\right.} \\
& \left.\times\left(\sqrt{1-\frac{y^{2}}{R_{\mathrm{j}}^{2}}} \cos \phi_{0}+\frac{y}{R_{\mathrm{j}}} \sin \phi_{0}\right)\right] \cos \beta .
\end{aligned}
$$

(ii) The far side edge of the jet (marked ii in Figs. 1 and 2; thick dashed line in Fig. 3). In this case the point is located at $(r, \sin \phi)=\left(R_{\mathrm{j}}, y / R_{\mathrm{j}}\right)$, with $\cos \phi=-\sqrt{1-y^{2} / R_{\mathrm{j}}^{2}}$. Substituting this value in Eq. (4) yields an expression like that in Eq. (6) for the jet velocity component along the line of sight, but with a minus sign in front of the square root.

(iii) The point closest to the jet's axis, i.e., on a line through the jet's axis and perpendicular to the line of sight (marked iii in Figs. 1 and 2; thin solid line in Fig. 3). This gas element resides at distance $y$ from the jet axis and perpendicular to the line of sight (i.e., in the $y z$ plane). In Eq. (4), substituting $r=|y|$ (see Fig. 2), and $\theta=\pi / 2$ and $\theta=3 \pi / 2$, for $y>0$ and $y<0$, respectively, gives

$$
\frac{\boldsymbol{v}_{\mathrm{j}}(r, \phi)}{v_{\mathrm{ja}}}=k \cos \beta-C_{1}\left[k+f_{2} \frac{y}{|y|} \sin \phi_{0}\right]\left(\frac{|y|}{R_{\mathrm{j}}}\right)^{n} \cos \beta .
$$

The following conclusions follow from Fig. 3, regarding the proposed scenario for jet-disk interaction.

1. This scenario explains the decline in radial velocity with increasing distance from the jet's axis. Such behavior is clearly observed in DG Tauri (Fig. 1 of B2002), in the red lobe of RW Aurigae (Fig. 5 of C2004), and in $\mathrm{LkH} \alpha 321$ (Fig. 5 of C2004).

2. An inclination of the jet to the outer disk with which it interacts (the inner disk that blows the jet $r_{\mathrm{d}} \lesssim 0.1-1 \mathrm{AU}$, is perpendicular to the jet) can explain the velocity shift between the two sides of each jet with the same sense of shift in the blue shifted (approaching) and red shifted (receding) jets.

3. Different regions along each line of sight have different projected velocities. The variation increases toward the jet's symmetry axis. If different spectral lines originate in different regions in the jet, then there will be large variations in the projected velocities between different spectral lines. This might be the case, for example, if instabilities in the jet-disk interaction region lead to formation of dense clumps, where the emission of some spectral lines are favored over other spectral lines. The instabilities might occur in some places, but not in others. Large differences in velocity profiles between different spectral lines are observed in a large fraction of the jets, e.g., in the red lobe of TH28 (Fig. 5 of C2004). The data are too noisy to tell whether the variation near the jet's center is larger.

4. Large velocity differences between the two jet's sides can be explained without invoking a huge amount of angular momentum in the jet.

\section{Summary}

The goal of the present paper is to provide an interpretation of the velocity profiles of some YSO jets without referring to any magnetic jet-launching model. These observations (B2002; C2004) show that the two sides of some YSO jets have different projected velocity (line of sight velocity), and that the sense of the velocity shift is the same in the blue (approaching) and red (receding) jets. B2002 and C2004 interpret the velocity profiles as rotation of the jets around their symmetry axis, something to be expected in some magnetic jet-launching models (Cerqueira $\&$ de Gouveia Dal Pino 2004).

In the Appendix I show that the angular momentum required to explain the rotation is at the upper limit of what the accretion disk can supply. In other words, to account for the required angular momentum and the kinetic energy of the jets, the mass outflow must be smaller than observed.

Alternatively, I propose a scenario where the velocity profile results from the interaction of the jets with the accretion disk. In Sect. 2 I argued that such an interaction is likely to 
occur, as accretion disks around YSOs might have a thick surface layer. I then (Sect. 3) presented a phenomenological scenario based on two assumptions. (1) There is an inclination between the jet and the disk further out. Namely, the inner disk is perpendicular to the jet, but then the disk flares in a pointsymmetric manner: one side up and the opposite side (relative to the central star) down (Fig. 2). (2) The disk slows the jet down, with larger decelaration of jet segments closer to the tilted disk and less efficient deceleration closer to the jets' axis. The velocity of the gas inside the jet as function of distance from the jet's symmetry axis $r$, and the angle relative to the direction where the disk flares up (in the $+z$ direction) $\phi-\phi_{0}$, was parameterized in Eq. (4). The different values appearing in that equation are defined in Figs. 1 and 2. The calculated projection velocity profiles for 4 cases are plotted in Fig. 3.

The conclusions drawn from Fig. 3 are summarized in the last paragraph of Sect. 3. In short, these are: (1) the proposed non-magnetic scenario explains the decline in radial velocity with increasing distance from the jet's axis. (2) The inclined jet-disk scenario might explain the velocity shift between the two sides of each jet with the same sense of shift in the blue shifted (approaching) and red shifted (receding) jets. (3) The large velocity variations between different spectral lines can be accounted for if different lines originate in different regions in the jets. For example, instabilities in the jet-disk interaction leads to formation of dense clumps where some lines are favored over others. (4) There is no need to depend on jet rotation with a huge amount of angular momentum.

The interaction of the warrped-tilted disk with the jet will cause a point-symmetric structure, and, high spatial resolution imaging may reveal such a structure close to the star. In Th 28, the H $\alpha$ image of the two HH objects possesses a pointsymmetric structure (Krauter 1986), which most likely comes from precessing jets. The two jets in RW Aur are highly symmetric and show no point-symmetry (Dougados et al. 2000). The one jet of DG Tau, as presented in the high velocity (HV) map by Lavalley-Fouquet et al. (2000; the upper row of their Fig. 1), possesses some wiggling. In any case, the predicted point-symmetry morphology is of small degree, but still might be noticed.

Another prediction of the proposed scenario is that in some cases, where disk changes twisting (wrapping) direction in a relatively short time period, the sense of asymmetry will change; i.e., the side which presently has a lower radial velocity, might slowly, say on a time scale of $\sim 100 \mathrm{yr}$, change to higher radial velocity. However, in most disks the expected time scale is very long, according to the different mechanisms that cause such twisting (or titling or warping; Armitage \& Pringle 1997; Larwood et al. 1996).

\section{References}

Anderson, J. M., Li, Z.-Y., Krasnopolsky, R., \& Blandford, R. D. 2003, ApJ, 590, L107

Anderson, J. M., Li, Z.-Y., Krasnopolsky, R., \& Blandford, R. D. 2005, ApJ, submitted [arXiv: astro-ph/0410704]
Armitage, P. J., \& Pringle, J. E. 1997, ApJ, 488, L47

Bacciotti, F., Ray, T. P., Mundt, R., Eislöffel, J., \& Solf, Jo. 2002, ApJ, 576, 222 (B2002)

Blandford, R. D., \& Payne, D. G. 1982, MNRAS, 199, 883

Bogovalov, S., \& Tsinganos, K. 2001, MNRAS, 325, 249

Canto, J., \& Raga, A. C. 1991, ApJ, 372, 646

Casse, F., \& Ferreira, J. 2000, A\&A, 361, 1178

Cerqueira, A. H., \& de Gouveia Dal Pino, E. M. 2004, A\&A, 426, L25

Coffey, D., Bacciotti, F., Woitas, J., Ray, T. P., \& Eisloffel, J. 2004, ApJ, 604, 758 (C2004)

Davis, C. J., Varricatt, W. P., Todd, S. P., \& Ramsay Howat, S. K. 2004, A\&A, 425, 981

Dougados, C., Cabrit, S., Lavalley, C., \& Menard, F. 2000, A\&A, 357, L61

Ferreira, J. 2002, in Star Formation and the Physics of Young Stars Summer school on Stellar Physics X, EAS Vol. III, ed. J. Bouvier, \& J.-P. Zahn (Les Ulis: EDP Sciences)

Ferreira, J., \& Casse, F. 2004, ApJ, 601, L139

Ferreira, J., \& Pelletier, G. 1993, A\&A, 276, 625

Ferreira, J., \& Pelletier, G. 1995, A\&A, 295, 807

Gaetz, T. J., Edgar, R. J., \& Chevalier, R. A. 1988, ApJ, 329, 927

Garcia, P. J. V., Ferreira, J., Cabrit, S., \& Binette, L. 2001, A\&A, 377, 589

Getman, K. V., Feigelson, E. D., Townsley, L., et al. 2002, ApJ, 575, 354

Heyvaerts, J., \& Norman, C. 1989, ApJ, 347, 1055

Heyvaerts, J., Priest, E. R., \& Bardou, A. 1996, ApJ, 473, 403

Kahn, F. D. 1980, A\&A, 83, 303

Kato, Y., Mineshige, S., \& Shibata, K. 2004, ApJ, 605, 307

Königl, \& Pudritz, R. E. 2000, in Protostars and Planets IV, ed. V. Mannings, A. P. Boss, \& S. S. Russell (Tucson: Univ. of Arizona Press), 759

Krasnopolsky, R., Li, Z.-Y., \& Blandford, R. D. 2003, ApJ, 595, 631

Krautter, J. 1986, A\&A, 161, 195

Larwood, J. D., Nelson, R. P., Papaloizou, J. C. B., \& Terquem, C. 1996, MNRAS, 282, 597

Lavalley-Fouquet, C., Cabrit, S., \& Dougados, C. 2000, A\&A, 356, L41

Li, L.-X. 2002, ApJ, 564, 108

Liseau, R., Fridlund, C. V. M., \& B. Larsson, B. 2005 ApJ, in press [arXiv: astro-ph/0410405]

Lynden-Bell, D. 2003, MNRAS, 341, 1360

Malbet, F., Lachaume, R., \& Monin, J.-L. 2001, A\&A, 379, 515

Pesenti, N., Dougados, C., Cabrit, S., et al. 2004, A\&A, 416, L9

Semenov, D., Wiebe, D., \& Henning, Th. 2004, A\&A, 417, 93

Shu, F. H., Lizano, S., Ruden, S., \& Najita, J. 1988, ApJ, 328, L19

Shu, F. H., Najita, J. R., Shang, H., \& Li, Z.-Y. 2000, in Protostars and Planets IV, ed. V. Mannings, A. P. Boss, \& S. S. Russell (Tucson: Univ. of Arizona Press), 789

Shu, F. H., Ruden, S. P., Lada, C. J., \& Lizano, S. 1991, ApJ, 370, L31

Soker, N., \& Lasota, J.-P. 2004, A\&A, 422, 1039

Soker, N., \& Regev, O. 2003, A\&A, 406, 603

Stahler, S. W. 1994, ApJ, 422, 616

Torbett, M. V. 1984, ApJ, 278, 318

Torbett, M. V., \& Gilden, D. L. 1992, A\&A, 256, 686 (TG)

Wijers, R. A. M. J., \& Pringle, J. E. 1999, MNRAS, 308, 207

Woitas, J., Bacciotti, F., Ray, T. P., et al. 2005, A\&A, 432, 149

Woitas, J., Ray, T. P., Bacciotti, F., Davis, C. J., \& Eisloffel, J. 2002, ApJ, 580, 336 


\section{Online Material}




\section{Appendix: Differential radial velocity along YSO jets}

In a recent paper for three YSOs, C2004 present observed profiles of line-of-sight velocity as function of the distance from the jet's symmetry axis. They interpret their observations as indicating jet rotation, and claim that the magnetocentrifugal jet launching model is consistent with their results. This is problematic on both accounts. More sophisticated and detailed MHD models for jet rotation, though, might overcome these problems. By examining the radial velocity figures of C2004, I note the following issues.

(1) Stochastic velocity profiles. Different lines show very different profiles, as is most evidence from their Fig. 5. In the red lobe of TH28, for example, the difference in velocity between the [NII] $\lambda 6548$ and the $[\mathrm{SII}] \lambda 6716$ is $\sim 30 \mathrm{~km} \mathrm{~s}^{-1}$ in the SW side, while the difference is almost an order of magnitude smaller in the NE side of the jet axis in the same lobe. In the YSO LkH $\alpha 321$ the difference between these two lines in the SE side reaches a value of $\sim 40 \mathrm{~km} \mathrm{~s}^{-1}$. These differences are larger than the claimed rotational velocity of the corresponding jets. Later, I attribute these differences between the velocities observed in different lines, and the difference between the two sides of the jets' axes, to the interaction of the jet with a tilted disk (Sect. 3).

(2) Angular momentum. If the radial velocity gradient is attributed to the jet's rotation, then the specific angular momentum of the gas at the edge of the jets must be extremely high. For example, in the redshifted lobe of RW Aur the toroidal velocities are $v_{\phi}=7-17 \mathrm{~km} \mathrm{~s}^{-1}$ (see last paragraph of Sect. 3 in C2004), in the range $y=14-28 \mathrm{AU}$, where $y$ is the distance from the jet's axis. The specific angular momentum is in the range $j \sim 100-500 \mathrm{AU} \mathrm{km} \mathrm{s}^{-1}$. For an accretion disk around a central object of mass $1 M_{\odot}$, this range corresponds to disk radii in the range $r_{\mathrm{d}} \sim 10-250 \mathrm{AU}$. In the magnetocentrifugal jet launching model the magnetic stress transports energy and angular momentum from the accreted mass to the ejected mass. In RW Aur the ejected mass is $\gtrsim 5 \%$ the accreted mass (Woitas et al. 2002), namely $\dot{M}_{\text {jet }} \gtrsim 0.05 \dot{M}_{\text {acc }}$, and C2004 find the foot point (where the streamline leaves the disk) of the outermost stream line to be at $r_{\mathrm{fp}}=1.3 \mathrm{AU}$. The inner-most foot point is at $r_{\mathrm{fp}}=0.4 \mathrm{AU}$. The specific angular momentum of Keplerian disk material at $r_{\mathrm{d}}=1.3 \mathrm{AU}$ is $\sim 0.07$ times the specific angular momentum of matter flowing on the outer stream line. This implies a very efficient angular momentum transfer. The accreted mass required to give all of its angular momentum is $\sim 14$ times the mass ejected in the outer parts of the jet (for $\dot{M}_{\text {jet }}=0.05 \dot{M}_{\text {acc }}$ ). For the average of the jet's specific angular momentum I take $j_{\mathrm{a}} \simeq 300 \mathrm{AU} \mathrm{km} \mathrm{s}{ }^{-1}$, which is also the value used in the numerical simulations of Cerqueira \& de Gouveia Dal Pino (2004). The total rate at which the jet carries angular momentum is then $\dot{J}_{\mathrm{j}}=\dot{M}_{\text {jet }} j_{\mathrm{d}}=15 \dot{M}_{\text {acc }} \mathrm{AU} \mathrm{km} \mathrm{s}{ }^{-1}$. In an undisturbed disk, the gas losses angular momentum as it flows from $r_{\mathrm{d}}=1.3 \mathrm{AU}$ to $r_{\mathrm{d}}=0.4 \mathrm{AU}$ (from outer to inner foot point) at a rate of $\dot{J}_{\mathrm{d}}=15 \dot{M}_{\text {acc }} \mathrm{AU} \mathrm{km} \mathrm{s}{ }^{-1}$. This implies that if C2004 interpretation of the jets in RW Aur is correct, then according to the jet-disk models they use, the disk must lose all of its angular momentum already at $r_{\mathrm{d}}=0.4 \mathrm{AU}$. This is a much larger radius than that of the accreting central star, which implies that the disk is truncated at a very large redius, where escape speed is much below the jet's speed.

(3) Energy. In the analysis of Anderson et al. (2003; see also 2005), the poloidal speeds along jets' stream lines of the YSO DG Tauri are $<3$ times what would be the escape velocity at the foot points of the stream lines. In the numerical calculations of Krasnopolsky et al. (2003) the poloidal terminal speeds along jet's streamlines are $\lesssim 2$ times the escape velocity at the foot point of the stream lines. The velocities found by C2004 (their Table 4) are much higher than the theoretical expectation; along most stream lines the terminal jets' speeds found by C2004 are $\gtrsim 3$ time the escape speeds. In the YSO LkH $\alpha 321$, for example, the jet speed is $\sim 4-4.5$ times the escape velocity. The required efficiency of energy transfer from accreted to ejected mass seems to be higher than that expected in the magnetocentrifugal models. Higher velocities are obtained in the numerical simulations of Garcia et al. (2001) which includes the thermal state of the jet. The major heating process of the jet is ambipolar diffusion. High poloidal velocities are obtained along stream lines, $\sim 10$ times the escape velocity from the footpoint of the stream line. However, the fraction of ejected mass (out of the accreted mass) is $1-2 \%$, much below the value of $\sim 10 \%$ in RW Tau (Woitas et al. 2005). But, again, in this section I am dealing mainly with C2004 interpretation of their observations, and not with the general MHD model.

(4) Model versus observations. C2004 use the jet launching model as presented by Anderson et al. (2003), who use it for the YSO DG Tau. However, in that model the toroidal velocity decreases with distance from the jet's axis, while in the observations, both of DG Tau (Anderson et al. 2003) and the three YSOs studied by C2004, the toroidal velocity increases with distance from the jet's axis. The opposite behavior is attributed by $\mathrm{C} 2004$ to projection and beam smearing effects. Still, despite these effects they compare their results with the theoretical model. I find this unjustified. In a recent paper Pesenti et al. (2004) present a more detailed MHD model based on jet rotation to account for the observation of DG Tau (B2002). Pesenti et al. (2004) find a good fit between their model and the velocity map of DG Tau. However, the model of Pesenti et al. (2004) for DG Tau has two significant differences from that of TW Aur (Woitas et al. 2005). First, their derive typical toroidal velocity for stream lines with footpoints of $\sim 1$ AU is much lower than that in TW Aur. In TW Aur the central star mass is $M_{*}=1.3 M_{\odot}$, and in DG Tau model of Pesenti et al. (2005) it is $M_{*}=0.5 M_{\odot}$. Velocities should be scaled like the square root of the central mass ratio $(1.3 / 0.5)^{1 / 2}=1.6$. Namely, the specific angular momentum in the outer jet radii in the model of Pesenti et al. (2004), of $\sim 80 \mathrm{AU} \mathrm{km} \mathrm{s}{ }^{-1}$, is much lower than that the value of $\sim 300-500 \mathrm{AU} \mathrm{km} \mathrm{s}{ }^{-1}$ found by 
Woitas et al. (2005) for the jets in TW Aur. The second difference involved the theoretical model used by Pesenti et al. (2004). Pesenti et al. (2004) find that only the warm jet model fit the observations. In this model the jet is thermally-driven (Casse \& Ferreira 2002). Both these differences, that the jets have low specific angular momentum, and are thermally driven, are along the main theme of the present paper, although significant differences exist between the warm MHD model used by Pesenti et al. (2004) and the model proposed here. 